\title{
Oxytocin Gene and Peptide Analysis in Children with Autism: A Preliminary Case Study
} \author{
Adolescent Psychiatry, Dayton, OH. \\ $\uparrow$ Corresponding Author \\ Wright State University \\ Boonshoft School of Medicine \\ Department of Pharmacology and Toxicology \\ Dayton, OH 45435-0001 \\ T: $937-775-2457$ \\ F: 937-775-7221 \\ david.cool@wright.edu
}

William C. Grunwald, Jr${ }^{1}$, Mariana Morris ${ }^{1}$, William M. Klykylo ${ }^{2}$ and David R. Cool $\uparrow^{1}$

Wright State University Boonshoft School of Medicine, ${ }^{1}$ Department of Pharmacology \& Toxicology, ${ }^{2}$ Department of Child and

\begin{abstract}
Autism is a neurodevelopmental disorder in which afflicted individuals exhibit characteristic impairments in social interaction, communication, cognition, imagination, and behavior. Though the root cause of autism is unknown, a defect in the oxytocin peptide hormone has previously been proposed to play a possible role in the development of autism. In this study we sequenced the oxytocin gene of two autistic children and eight non-autistic control individuals. There was a lack of sequence polymorphism within the three exons of the oxytocin gene from patient samples and the control group. SELDI-TOF mass spectrometric analysis of the oxytocin peptide in the plasma revealed the presence of mature oxytocin. Other forms or extended forms of oxytocin were not observed. We conclude that in these patients, defects within the oxytocin peptide do not play a role in autism.
\end{abstract}

Keywords-autism; oxytocin; SELDI-TOF mass spectrometry; gene analysis 


\subsection{Introduction:}

Autism is a developmental disorder manifested in impaired communication skills and abnormal social interactions (Wing and Gould 1979, Schopler et al. 1980, Wing 1997, Insel 1997, Cook et al. 1998). There are also obvious defects in cognition, behavior and imagination (Wing and Gould 1979). Autism is a chronic disorder that usually develops before 36 months of age (Bailey and Rutter 1991). Investigations and understandings of the condition have come a long way since Kanner first described the disorder in 1943 (Kanner 1943). Initial observations clearly separated autism from other psychiatric disorders with descriptions of peculiar language usage, repetitive behavioral patterns and the apparent inability to relate to people (Kanner 1943). Through studies involving twins, a polygenic link to the autistic disorder was made that does not appear to follow Mendelian patterns of inheritance (Pickles et al. 1995) suggesting that more than one gene may be involved in autism. One candidate gene product known to play a role in behavior that has been proposed to play a role in autism is oxytocin (Insel and Shapiro 1992, Insel, O'Brien, and Leckman 1999).

Oxytocin is a peptide hormone that is synthesized in the hypothalamus and secreted into the bloodstream from the posterior pituitary and plays a role in many peripheral processes, such as uterine contractile response, parturition and milk letdown (Brownstein 1983, Land et al. 1983, Nishioka et al. 1998, Samson, Lumpkin, and Mccann 1986, Akerlund 1993). However, oxytocin is also released centrally to other regions of the brain where it can interact with receptors to influence sexual behavior, social interaction and other behavioral processes (Carter 1992, Insel 1992, Witt 1995, van Wimersma Greidanus and Maigret 1996a, Barberis and Tribollet 1996, Insel and Shapiro 1992, van Wimersma Greidanus and Maigret 1996b, Young et al. 2001). To study whether oxytocin plays a role in autism, blood plasma oxytocin levels were measured in autistic children and normal controls (Modahl et al. 1998). The results suggested that the ratio of mature oxytocin to "extended" forms of immature oxytocin was nearly double that found in control patients (Green et al. 2001). Given certain ubiquitous phenotypes of the autistic disorder relating to the lack of social attachment, defects in the oxytocin gene structure, expression or processing mechanisms seem a likely candidate for investigation.

In this study, we sequenced the oxytocin gene from two patients and 8 control subjects to determine if there is a defect that might cause aberrant processing of oxytocin in some autistic children. We report that there are no single nucleotide polymorphisms (SNPS) in the exons of the oxytocin gene in these patients. In addition, Surface Enhanced Laser Desorption Ionization-Time-Of-Flight Mass Spectroscopy (SELDI-TOF MS) was used to analyze the oxytocin peptide in the patient samples.

\subsection{Materials and Methods: 2.1 Patients}

Two autistic patients and eight non-autistic controls were selected for this study. The male patients were 8 yrs 9 months and 11 yrs old and met the current diagnostic criteria established in the Diagnostic and Statistical Manual of Mental Disorders-IV (American Psychiatric Association, 1994 DSM-IV) for a diagnosis of autism.

\subsection{PCR Analysis of Patient Blood for the Oxytocin Gene}

Approximately $5 \mathrm{mls}$ of blood was collected in heparinized tubes in accordance with WSU-IRB guidelines. Whole blood samples consisting of $2 \mathrm{mls}$ each were used in the DNA isolations of all patient and control samples. All genomic DNA isolations were performed with Qiagen's QIAamp ${ }^{\circledR}$ DNA blood midi kit (Valencia, CA). The oxytocin gene amplifications were carried out with the use of the FailSafe ${ }^{\mathrm{TM}}$ PCR PreMix Selection Kit (Epicentre Technologies, Madison, WI). Conditions for PCR reactions were as follows: $25 \mu \mathrm{l}$ FailSafe $^{\mathrm{TM}}$ PCR 2x Premix D, $0.5 \mu \mathrm{l}$ FailSafe $^{\mathrm{TM}}$ PCR enzyme mix, $1 \mu \mathrm{l}$ primer OTE1 (5'CAGAGCTCCACCGACGCAATG-3'; $(0.125 \mu \mathrm{g} / \mu \mathrm{l}), 1 \mu \mathrm{l}$ primer OTE3 (5'-CTGGGGTGGCTATGGGGGAAG-3'; $(0.125 \mu \mathrm{g} / \mu \mathrm{l})$, $1 \mu \mathrm{l}$ whole genomic DNA $(0.05 \mu \mathrm{g} / \mu \mathrm{l})$, and $21.5 \mu \mathrm{l}$ of distilled dionized water for a total of a $50 \mu \mathrm{l}$ reaction volume. PCR cycling conditions: $96^{\circ} \mathrm{C}$ for five minutes, $96^{\circ} \mathrm{C}$ for one minute, $60^{\circ} \mathrm{C}$ for one minute, $68^{\circ} \mathrm{C}$ for one minute for thirty-two cycles, and finally $72^{\circ} \mathrm{C}$ for seven minutes on a Bio-Rad iCycler PCR machine (Bio-Rad, Hercules, CA). All PCR products were separated on $1.5 \%$ agarose gels stained with ethidium bromide and visualized by UV transillumination. All PCR products were purified with Qiagen's QIAquick ${ }^{\circledR}$ PCR purification kit and then sequenced using all four primers, i.e., OTE1, OTE1.2, OTE2, OTE3 (Lark Technologies, Inc., Houston, TX; GenomatixUSA, Cincinnati, OH). Sequence data was aligned with the use of MultiAlign, an internet driven multiple alignment program (Corpet 1988) and compared with the GenBank sequence for oxytocin, accession \#M11186 (Sausville, Carney, and Battey 1985). The concensus DNA sequence derived from this data has been deposited on GenBank, accession \#AY082910.

\subsection{C18SepPak Peptide Extraction}

Peptides and proteins were extracted from plasma using SepPak C18 columns (Cool and DeBrosse 2003). SepPak (Millipore) C18 columns were conditioned with $3 \mathrm{ml}$ methanol, followed by $3 \mathrm{ml}$ of distilled water. One $\mathrm{ml}$ of patient plasma was passed through the columns. The columns were washed with 3 mls of water followed by $3 \mathrm{mls}$ of $3 \%$ acetone and $1 \mathrm{ml}$ fractions collected. The peptides were eluted by $3 \mathrm{ml}$ of $80 \%$ acetonitrile containing $0.1 \%$ TFA (vol/vol) and $1 \mathrm{ml}$ fractions were collected. All samples were dehydrated on a Savant Speed Vac and reconstituted in $0.2 \mathrm{ml}$ of RIA buffer. For SELDI-TOF MS, another $\mathrm{ml}$ of the plasma was extracted by the same method, dried and resuspended in $20 \mu \mathrm{l}$ of $0.1 \mathrm{~N} \mathrm{HCl}$.

2.4 Ciphergen ProteinChip ${ }^{\circledR}$ SELDI-TOF Mass Spectrometry Weak cation exchange (WCX2) ProteinChips ${ }^{\circledR}$ (Ciphergen, Palo Alto, CA) were spotted with peptides from; 1) rehydrated 
oxytocin and AVP standards (10 $\mathrm{ng} / \mu \mathrm{l})$; and 2) plasma from two autistic male patients that had been extracted on C18-SepPak columns (Cool and DeBrosse 2003). One microliter of each sample was spotted onto the WCX2 ProteinChip ${ }^{\circledR}$ and washed with $5 \mu$ distilled water. Matrix, alpha-cyano-4-hydroxy cinnamic acid (CHCA) in 50\% acetonitrile containing $0.1 \%$ TFA $(0.5 \mu \mathrm{l})$ was added to the spots and allowed to dry. The ProteinChips ${ }^{\circledR}$ were analyzed on a Ciphergen SELDI ${ }^{\mathrm{TM}}$ Protein Biology System II (Ciphergen, Palo Alto, CA) with a spot protocol that ionized each spot four times over twenty different areas. The spot was initially ionized with 1 laser hit at 200 intensity, the sensitivity set at 10, followed by 4 laser hits at 195 intensity. The source voltage was set at 20,000 volts and detector voltage at 1,900 volts. The 80 laser hits were averaged for each spot and the resulting protein profile analyzed to determine the mass/charge of each molecular ion species.

Figure 1

A

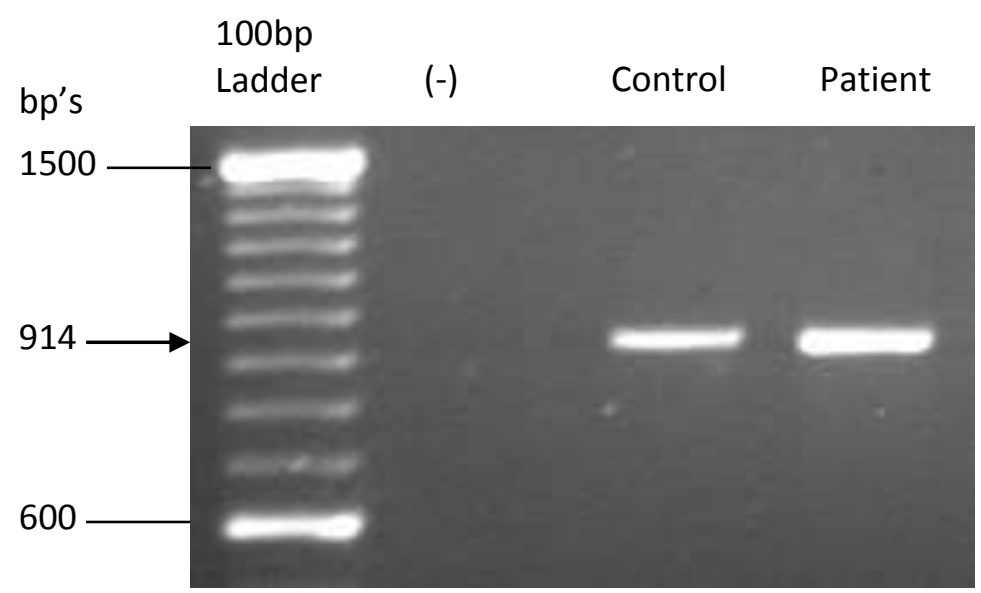

B

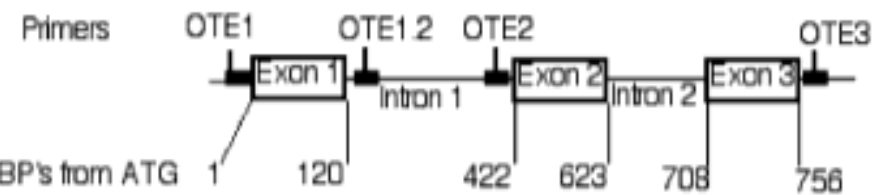

Figure 1. Agarose gel of PCR products showing size and purity of the reaction (A). The PCR products from a single patient and control were run on $1.5 \%$ agarose gel and visualized by UV illumination. The single band observed was approximately 914 bp's in length as determined by comparison with the $100 \mathrm{bp}$ ladder. The first lane (-) represents the negative control in which genomic DNA was not added to the reaction. B) Schematic map of the oxytocin gene. The bp's indicate the beginning and end of exons relative to the ATG start codon. Primers OTE1 and OTE3 were used to generate the full-length oxytocin PCR product. Primers OTE1, OTE1.2, OTE2 and OTE3 were used for forward and reverse sequencing of the PCR product.
3.0 Results:

\subsection{Exon Analysis}

Figure 1A shows PCR amplification products of the oxytocin gene in the target range $(914 \mathrm{bp})$ for an autistic patient and a control individual. These bands contain three exons and two introns that make up the oxytocin gene and were sequenced using two sets of forward and reverse primers (Figure 1B). The second exon and first intron contained differences when compared against the GenBank sequence for the human oxytocin gene M11186. In exon 2, both the patient and control consensus sequences show a silent $\mathrm{G}$ to $\mathrm{C}$ substitution at nucleotide position 597 compared with the GenBank reference sequence, accession \#M11186 (Figure 2a). Immediately downstream from this, a GTC that codes for a valine residue was inserted into the sequence, at nucleotide position 598, 599 and 600 (Figure 2b). This sequence was not present in the reference M11186 sequence, but was predicted as being present by amino acid sequencing of the prohormone (Chauvet et al. 1983). The sequences are shown for these differences in Figure $3 \mathrm{a}$ and $\mathrm{b}$. 
Figure 2

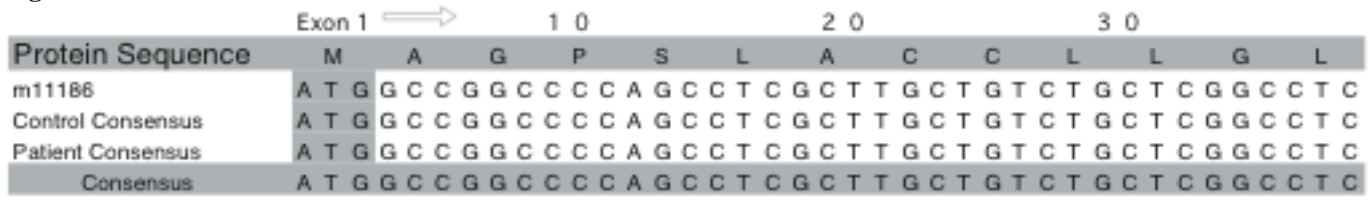

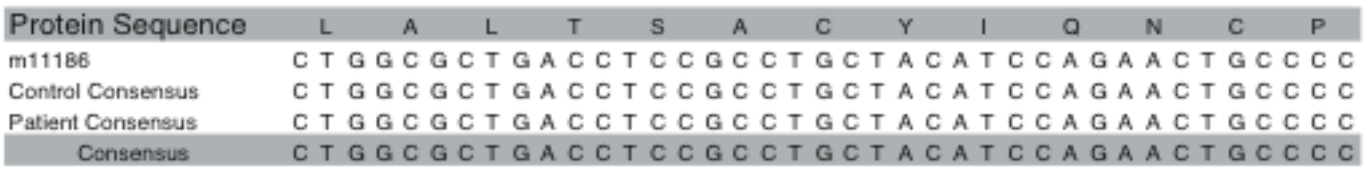

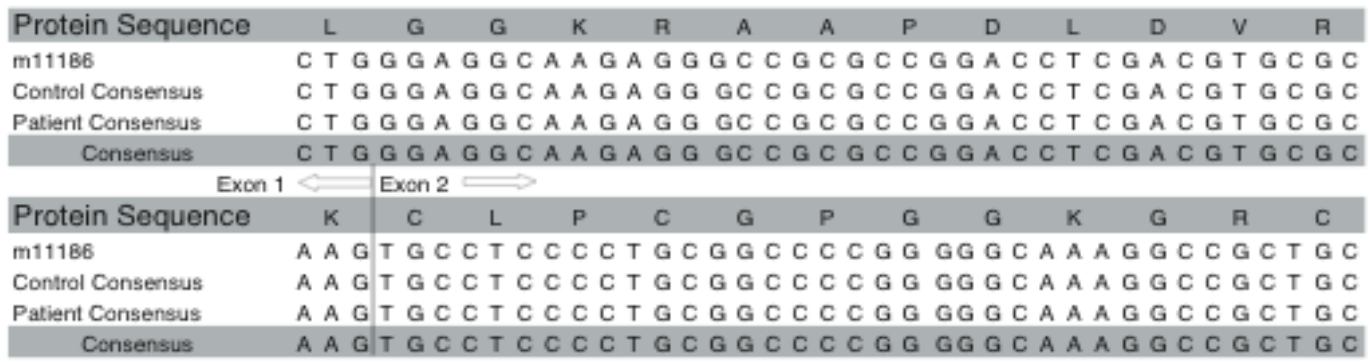

\begin{tabular}{|c|c|c|c|c|c|c|c|c|c|c|c|c|c|c|}
\hline rotein S & $\mathbf{F}$ & G & $\mathrm{P}$ & $\mathrm{N}$ & 1 & & C & C & A & $\mathrm{E}$ & E & L & G & C \\
\hline 11 & $T T$ & G C & C & A A T & A T C & C T C & GCT & $c$ & 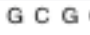 & & GA G & $\therefore 9$ & 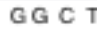 & $I_{G C}$ \\
\hline sus & $T T$ & G G & C & A A T & A T C & C T C & GCT & & G C & G A & & $T G$ & $c$ & 16 \\
\hline atient C & $T T$ & G G & $\mathrm{C} \mathrm{C} \mathrm{C}$ & A A $T$ & A T C & & G C T & c & G C & G & GA G C & $T G$ & GG C T & G C \\
\hline is & $T T$ & 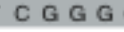 & C & A A $T$ & A T C & & $=1$ & & 3 & GA A & GA & T G & GCT & i $\mathrm{C}$ \\
\hline
\end{tabular}

\begin{tabular}{|c|c|c|c|c|c|c|c|c|c|c|c|c|c|c|}
\hline Protein Sequence & $\mathbf{F}$ & & $\mathrm{v}$ & G & $T$ & A & $\mathrm{E}$ & A & L & R & $c$ & $a$ & E & E \\
\hline 11186 & T & & $c$ & U & & & GA & & & C G & & & & \\
\hline ontrol Co & $T T$ & $G$ & $T G C$ & G G & & $\mathrm{C}$ & $C G A$ & (3) & & $c^{2}+2 x$ & & & & $A G$ \\
\hline atient C & $T T$ & C G & Go & $\mathrm{GC}$ & $\mathrm{C}$ & GC & CGA & $\mathrm{GC}$ & C T & GCGCT & $=$ & $A G G$ & U & G \\
\hline Consensus & & & & & & C & & & C T & C T & C C & & a G & \\
\hline
\end{tabular}

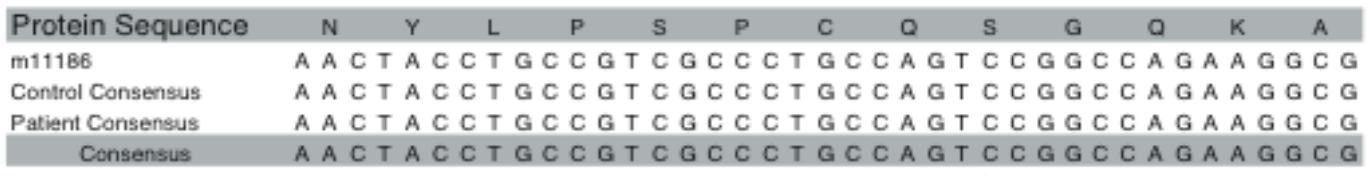

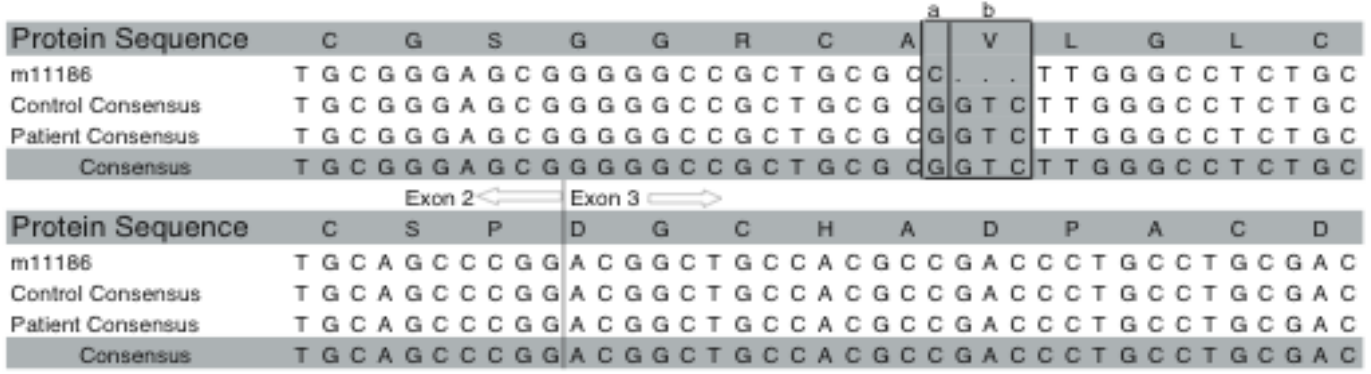

\begin{tabular}{|c|c|c|c|c|c|c|c|c|c|}
\hline Protein Sequence & A & E & A & $T$ & $\mathrm{~F}$ & s & Q & A & OPAL \\
\hline m111 & G C & A & $\mathrm{C}$ & C C T & $T C T$ & $\mathrm{C} \mathrm{C}$ & $A G$ & G C & $\mathrm{TCC}$ \\
\hline Control Consensus & G C & A A & G C & $A \subset C T$ & $T C T$ & $\mathrm{C} \mathrm{C}$ & $A G C$ & G C & $T G A$ \\
\hline Patient Consensus & G C & G A A & G C C & $A \subset C T$ & $T C T$ & & & & $T \in A$ \\
\hline $\mathrm{Co}$ & & & & & & & & $c$ & 1 \\
\hline
\end{tabular}

Figure 2. DNA and protein sequence of the three oxytocin exon coding regions. The top line is the single amino acid code derived from the DNA sequence. M11186 is the accession number for the GenBank reference sequence used for comparison of the control and patient sequences. Since there was no difference in the patient and control sequences, we chose to display the sequence results as a consensus. The final consensus sequence was determined from both the control and patient sequences. Variations from the M11186 sequence are labeled a \& b. 
Figure 3

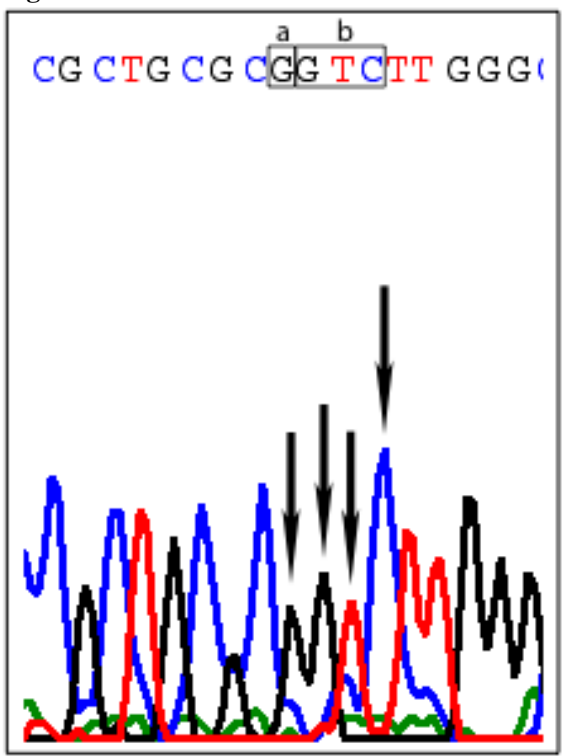

Figure 3. Autosequence chromatogram for the sequence variations identified in figure 2 . Boxed areas and arrows indicate the variance from the M11186 consensus sequence.

\subsection{Intron Analysis}

There were four regions in intron 1 that contained nucleotide changes with respect to the GenBank reference sequence, M11186. All four variances with respect to M11186 were conserved between patient and control sequences. There was a CG inserted after nucleotide position 277 for all patient and control sequences that was not present in the M11186 reference sequence (Fig. 4a \& 5a). At nucleotide position 298 (Fig4b \& 5b), the M11186 sequence had a C that was not found in control or patient samples. The last two differences were found at nucleotide positions 393 and 394 (Fig4c \& 5c) and 418 and 419 (Fig4d \& 5d) where there were CG to GC flips.

An alignment of the intron 2 sequence data revealed no differences between the M11186 GenBank reference sequence and our patient and control consensus

\section{Figure 4}

m11186

Control Concsensus

Patient Concensus

Consensus

GT GA GT C C C CA GCCCT GGTCCCGCGGCGCTCCGGGGAGG GT GAGTCCCCAGCCCTGGTCCCGCGGCGCTCCGGGGAGG GT GAGTCCCCAGCCCT GGTCCCGCGGCGCTCCGGGGAGG

m11186 Control Concsensus GAGGGACCCGCAGCCACAGGGGCGCGCCCCGCT C CGGCC Patient Concensus GAGGGACCCGCAGCCACAGGGGCGCGCCCCGCTCCGGCC Consensus GAGGGACCCGCAGCCACAGGGGCGCGCCCCGCTCCGGCC

m11186 TCGCCTGAGAACTCCAGGAGCTGAGCGGATTTTGACGCC Control Concsensus TCGCCTGAGAACT CCAGGAGCTGAGCGGAT T T T GACGCC Patient Concensus TCGCCTGAGAACTCCAGGAGCTGAGCGGAT T T T GACGCC Consensus TCGCCTGAGAACTCCAGGAGCTGAGCGGATT T T GACGCC

m11186 CCGCCCTTGACCGCGGTCGAGGCCCCCACGGCGCCCCAG Control Concsensus CCGCCCTTGACCGCGGTCGAGGCCCCCACGGCGCCCCAG Patient Concensus CCGCCCTTGACCGCGGTCGAGGCCCCCACGGCGCCCCAG Consensus $\quad \mathrm{CCGCCCTTGACCGCGGTCGAGGCCCCCACGGCGCCCCAG}$ m11186 CG. T T TCAGCCCCGCTGTCCO Control Concsensus CGCGTCTCAGCCCCGCTGTCCO. GCCCGAACTCCGAACC Patient Concensus CGCGTCTCAGCCCCGCTGTCCO. GCCCGAACT CCGAACC Consensus $\quad$ GCGTCTCAGCCCCGCTGTCCD. GCCCGAACT CCGAACC

m11186 CCGGACCCCAGCATCCTTGCCCGGCGCACCCCGGCCGGC Control Concsensus CCGGACCCCAGCATCCT T GCCCGGCGCACCCCGGCCGGC Patient Concensus Consensus CCGGACCCCAGCATCCTTGCCCGGCGCACCCCGGCCGGC CCGGACCCCAGCATCCT T GCCCGGCGCACCCCGGCCGGC

m11186

CTCGCAGGGTCCTCCGAGCGAGTCCCCAGCGCCGCCCCG Control Concsensus CTCGCAGGGTCCT C CGAGCGAGT C C C CAGCGCCGCCCCG Patient Concensus CTCGCAGGGTCCTCCGAGCGAGTCCCCAGCGCCGCCCCG Consensus CTCGCAGGGTCCTCCGAGCGAGT CCCCAGCGCCGCCCCG m11186 CGTCCCGCTCACCCCGCCCGTCCCO Control Concsensus GOT CCCGCT CACCCCGCCCGT C CCOGOAG Patient Concensus GOT CCCGCTCACCCCGCCCGTCCCOGOAG Consensus GOTCCCGCTCACCCCGCCCGTCCCOGOAG

Figure 4. DNA sequence of intron 1. Patient and control sequence variations compared to M11186 are indicated by boxed bases under labels a-d. 
Figure 5

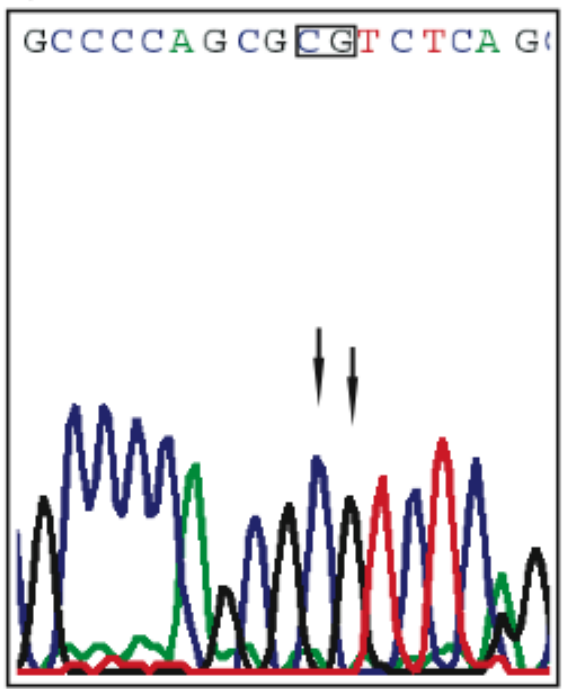

C

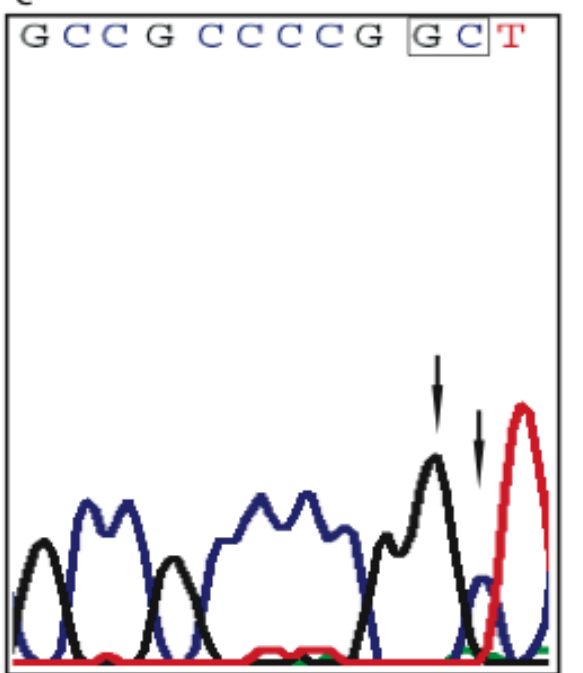

Figure 5. Autosequence chromatogram for the sequence variations identified in Figure 4. Boxed

areas and arrows indicate variations from M11186.

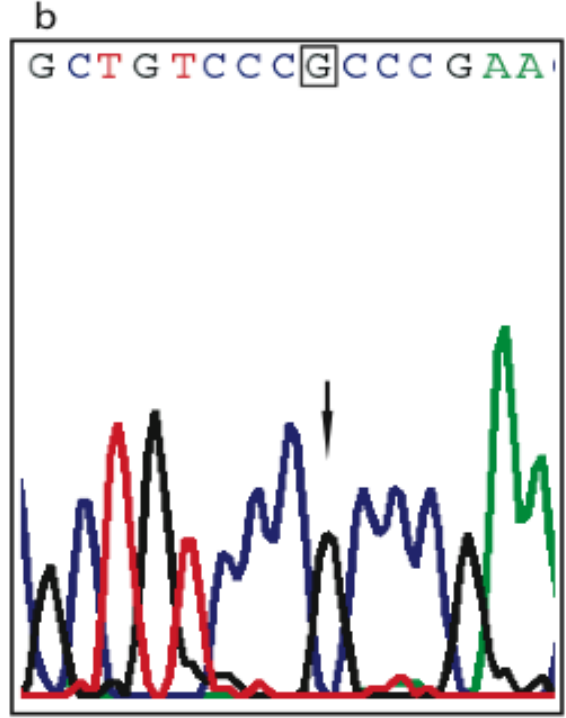

d

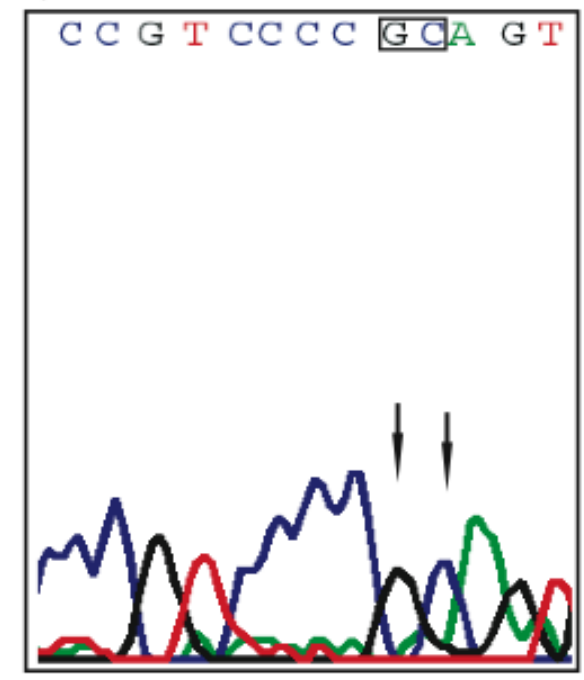

\subsection{Analysis of Oxytocin Peptide by Radioimmunoassay}

In previous reports, plasma oxytocin levels in autistic children were reported to be lower than in control children (Modahl et al. 1998, Green et al. 2001). The plasma levels of oxytocin peptide in both autistic patients were determined by radioimmunoassay. Plasma was extracted using a C18SepPak and peptides eluted from the column with $80 \%$ acetonitrile (Cool and DeBrosse 2003). Oxytocin concentrations were 4.15 picograms/milliliter for patient 1 (Cool and DeBrosse 2003) and 16.2 picograms/milliliter for patient 2 . These levels are higher than previously reported for both autistic or control groups (Modahl et al. 1998, Green et al. 2001).

\subsection{SELDI-TOF MS Analysis of Oxytocin}

Surface Enhanced Laser

Desorption/Ionization-Time-Of-Flight

Mass Spectrometry is a relatively new technique in which proteins and peptides are applied to chemically treated ProteinChips ${ }^{\circledR}$ and then selectively retained or removed from the ProteinChips ${ }^{\circledR}$ by washing (Hutchens and Yip 1993, Merchant and Weinberger 2000, Issaq et al. 2002, Wright et al. 1999). In a previous investigation of autistic individuals, the oxytocin peptide was proposed to be of an extended form, as determined by antibody-specific RIA, though the exact nature of the extended form was not characterized (Green et al. 2001). To determine if oxytocin was in an extended form in the autistic patient samples, the C18-SepPak eluates were analyzed by SELDI-TOF MS and molecular ion peaks corresponding to oxytocin and AVP were identified (Figure 6A, C and D). The main peptides found in the range of oxytocin were; oxytocin (1,009.22 Daltons) and AVP (1,084.21 Daltons). C-terminally extended forms of oxytocin ions, i.e., oxytocin-Gly, oxytocin-Gly-Lys or 
oxytocin-Gly-Lys-Arg (1,066, 1,194 and 1,350 Daltons, respectively) were not identified in any of the samples. Though not directly related to oxytocin, extended forms of AVP were not observed in either patient. Standards for oxytocin, extended-form oxytocin and AVP (Figure 6A \& B) were used to calibrate the SELDI-TOF MS profiles obtained with the patient sample (Figure 6 C). The SELDITOF MS profile from patient two showed similar results (Cool, 2002).

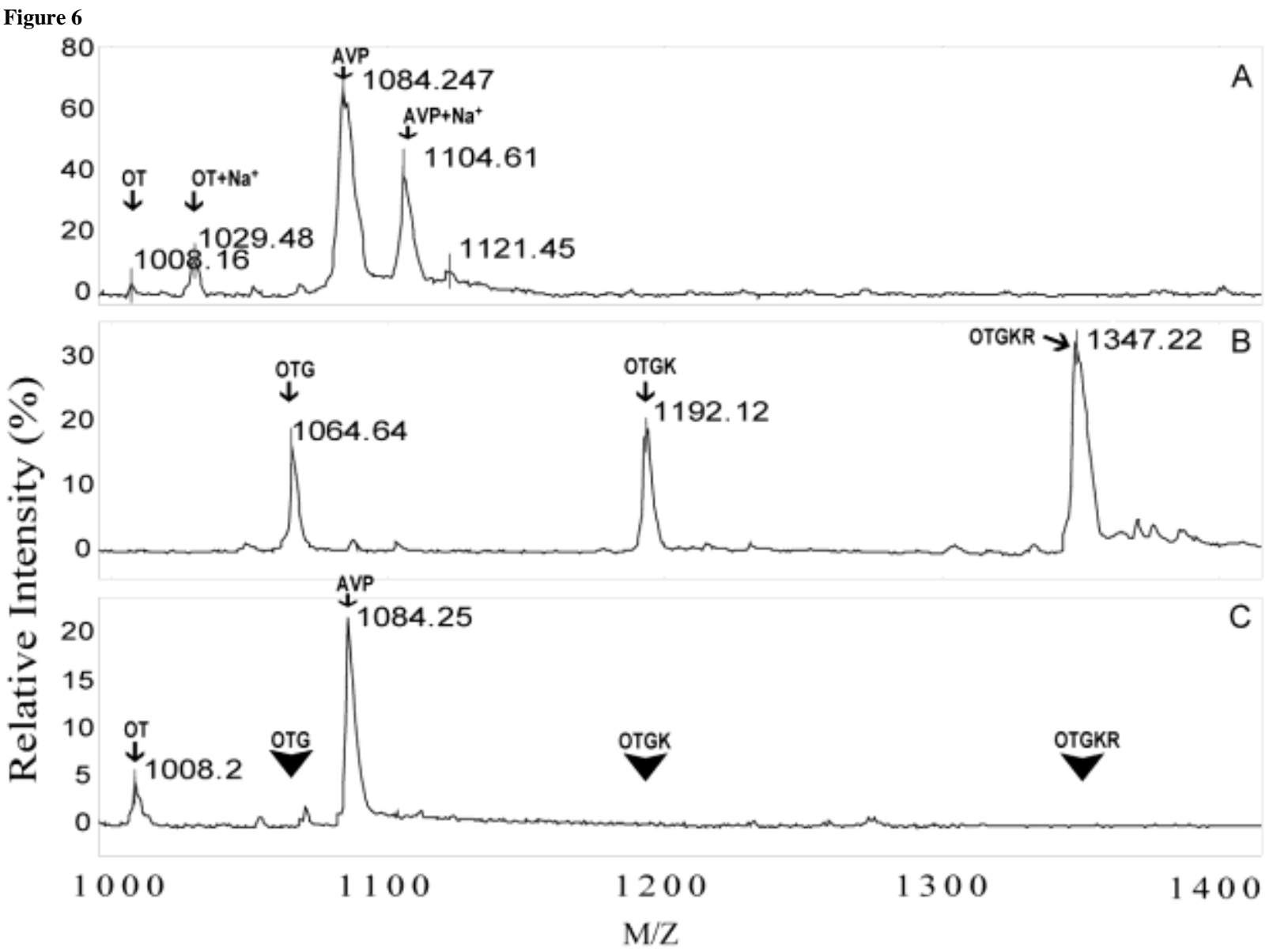

Figure 6. Analysis of autistic patient plasma by SELDI-TOF Mass Spectromtry. A) Ten nanograms of oxytocin and AVP peptide standards were applied to a WCX2 (Weak Cation Exchange) ProteinChip ${ }^{\circledR}$ and analyzed by SELDI-TOF MS. Oxytocin $(\mathrm{OT})$, oxytocin + sodium $\left(\mathrm{OT}+\mathrm{Na}{ }^{+}\right), \mathrm{AVP}$ and $\mathrm{AVP}+$ sodium $\left(\mathrm{AVP}+\mathrm{Na}^{+}\right)$are indicated by arrows. B) Ten nanograms of extended forms of oxytocin, i.e., OTG (oxytocin-glycine), OTGK (oxytocin-glycine-lysine) and OTGKR (oxytocin-glycine-lysine-arginine) are indicated by arrows. Plasma from autistic patient 1 (C) was extracted using C18 SepPaks and analyzed by SELDI-TOF MS. Arrowheads indicate the positions of expected C-terminally extended forms of oxytocin.

\subsection{Discussion}

In this study, several differences were found in the sequence alignments of the oxytocin gene compared to the GenBank sequence used as a reference, M11186. The first variance was a silent $\mathrm{C}$ to $\mathrm{G}$ conversion in exon 2 . The second variance, also in exon 2, was a three nucleotide insertion that codes for valine and was not found in the reference sequence. However, in an earlier study, amino acid sequencing showed that the valine did exist (Chauvet et al. 1983). Comparison to the chromosome 20p13 sequence, generated through the human genome project confirms that the sequence and valine are present. These two sequence variations are not SNP's and thus should have no impact on the sorting, processing or secretion of oxytocin from the pituitary.

In intron one, four conserved sequence variations were observed. This suggests that the original M11186 sequence either was not correct at each of the positions or there were numerous SNP's in the coding and non-coding regions of the DNA from the person donating the sample for the M11186 reference. None of the variances we have identified created or destroyed any of the GT-AG splice sites. Thus, the four variances located in intron 1 should not be involved in changing the mRNA sequence coding for oxytocin. The consensus sequence reflecting changes in the 
gene sequence have been published in GenBank, accession \#AY082910.

Does oxytocin play a role in autism? In a previous study, variations in blood levels of oxytocin were described (Modahl et al. 1998). A second study showed that the total blood levels of oxytocin did not change appreciably, however, there were putative changes in the processed and 'extended forms' of oxytocin (Green et al., 2001). This could occur as a result of; 1) polymorphisms in the prohormone causing misfolding of the prohormone and incorrect processing; 2) inactivity of the processing enzymes; or 3) variation in the degradative enzymes in the blood. The results from our study showed no evidence of 'extended forms' of oxytocin, i.e., OTGK or OTGKR. The differences in our results and the previous two studies could be explained by the use of antibodies with overlapping specificity (Green, 2001) versus mass spectrometry that can differentiate the exact identity of each of these peptides. In the Green study, antibodies to OT and OT-GKR were used that relied on specificity based on the two amino acid difference between 9 amino acid OT and 11 amino acid extended OT-GKR, i.e., a lysine and arginine residue. The authors suggested an overlap between the two antibodies but provided no description about how this antibody overlap was accounted for or subtracted to yield the final results (Green et al., 2001). Furthermore, variability introduced by running samples at different times, loss of peptide in the acetone:ether extractions, and the differential sensitivity of the antibodies could also explain some of the differences. In contrast, mass spectrometry provides the observed mass of the peptides that can be compared with the theoretical mass of the peptides. Thus, the identity of the peptide is not the result of differential antibody specificity but rather is a direct correlation to the mass of the peptide. Furthermore, the preparation of samples for mass spectrometry is a more direct route to sample analysis with less peptide loss, i.e., SepPak preparation of the samples, concentration in a speed vac and analysis on the mass spectrometer. In a previous comparison of these two sample preparations, we found that the SepPak method provided more consistent and efficient peptide recovery than acetone:ether precipitation (Cool and DeBrosse, 2003). One additional point, the addition of charged amino acids, i.e, lys and arg, to a fairly neutral peptide such as oxytocin would cause an increased ionization of the peptide which would lead to increased sensitivity for the extended forms of oxytocin. The lack of an ion peak at any of the expected masses determined for the extended forms provide strong and direct evidence that OTG, OTGK and OTGKR were not in the blood of the patient in this study nor in the blood of the patient in our first study (Cool \& DeBrosse, 2003).

In the present study, sequencing the oxytocin gene from an autistic patient and control subjects revealed no polymorphisms in the exon coding regions or introns that would suggest a defect in the oxytocin peptide in autistic patients. Although SNPs or larger scale mutations could exist in the oxytocin genes in autistic children, it is probable that these would be present in only a small subset of patients. Therefore, it is worthwhile to investigate other mechanisms that could explain the previously reported aberrant levels more thoroughly.

If the oxytocin gene is not a factor in these patients, then mutational analysis of the processing enzymes should be considered, as this would be more likely to yield 'extended forms' of the peptide hormone. There are several processing steps that must occur in the maturation of a peptide hormone such as oxytocin. The first processing step is cleavage of the prohormone at paired-basic residues to release the "extended form" of the peptide hormone containing a pair of basic residues at the $\mathrm{C}$ terminus (Steiner et al. 1992, Lindberg 1991, Seidah et al. 1990). In the second of the two studies that showed variances in blood oxytocin levels, autistic patients were found to have higher levels of the extended forms of oxytocin (Green et al. 2001). This suggested that the primary processing step involving PC2 was probably not defective. Following initial cleavage by $\mathrm{PC} 2$, a second processing step involving the enzyme, carboxypeptidase $\mathrm{E}$ (CPE), is necessary to remove the basic residues from the peptide (Fricker and Snyder 1983, Fricker 1988, Fricker 1991, Hook 1985). Variations in the CPE gene have not been identified in past studies of human patients (Utsunomiya et al. 1998). However, a mouse model has been characterized that has a spontaneous defect, substitution of a single amino acid, in the CPE gene that results in inactive CPE protein (Naggert et al. 1995, Fricker et al. 1996, Varlamov, Leiter, and Fricker 1996). The $\mathrm{CPE}^{\mathrm{fat}}$ mice expressing this defective gene had increased levels of pro-insulin in the blood, i.e., hyperproinsulinemia (Naggert et al. 1995, Fricker et al. 1996, Varlamov, Leiter, and Fricker 1996). In the present study, SELDI-TOF MS was used to determine if extended forms of oxytocin were present in the plasma from the autistic patients. The lack of C-terminally extended forms of oxytocin ions, i.e., Gly, Gly-Lys or Gly-Lys-Arg, suggests that in these patients, processing of oxytocin was not altered. Furthermore, if CPE was in fact found to be defective or deficient in autistic patients or a subset of these patients, then the autistic children would be expected to have multiple peptide hormone defects. This has not been described in depth for these patients or other autistic patients. Although the oxytocin levels in the present study were higher than those reported in previous studies, i.e., $\sim 4$ and $16 \mathrm{pg} / \mathrm{ml}$ vs $0.5-3 \mathrm{pg} / \mathrm{ml}$ (Modahl et al. 1998), variations in the oxytocin peptides that are defined as extended forms could represent minor peptides that are not concentrated enough to be observed by SELDI-TOF MS. In addition, since the sample number in this case study is small, extended forms of oxytocin may be present in other patients, and certainly, as part of a comprehensive study, should be determine for each patient.

Previous research on prairie and Montane voles provided the impetus for studies on oxytocin and AVP in behavioral disorders such as autism (Carter 1992, Insel 1992, Witt 1995, van Wimersma Greidanus and Maigret 1996a, Barberis and Tribollet 1996, Insel and Shapiro 1992, van Wimersma Greidanus and Maigret 1996b, Young et al. 2001). In these studies, oxytocin receptor distribution was found to be different between the two types of voles. This differential receptor expression was correlated with opposite behavioral and social characteristics, 
e.g., pup-rearing, maternal instinct, male-female bonding, etc. increasingly unlikely from the current study that polymorphisms Further links between oxytocin and vasopressin were identified in the oxytocin gene contribute directly to the heterogeneity and when the promoter regions of these receptors were compared broad spectrum of the autistic disorder. Thus, future studies between the prairie and Montane voles (Young 2001). should focus on each of the peptide hormone synthesis steps Polymorphisms in the number of tetranucleotide repetitive outlined above, beginning with carboxypeptidase E, as well as elements in the promoter regions have been identified in humans. polymorphisms in and distribution of brain oxytocin receptors.

The implication is that non-coding regions of receptor genes may play a role in the tissue-specific expression of the oxytocin and AVP receptors, which could lead to variability in behavior. This then strongly suggests that the sequences of oxytocin and AVP receptor genes and promoter regions should be analyzed for polymorphisms that could help explain the autistic disorder.

Autism is a complex disorder that potentially has many

\subsection{Acknowledgments}

This research was funded by grants from the National Alliance for Autism Research, the National Institutes of Health (1R01 DK58111-02) and from the Department of Defense (DAMD17-00-C-0020).

different causes, both genetic and environmental. It seems 


\subsection{References}

Akerlund, M. 1993. "The role of oxytocin and vasopressin in the initiation of preterm and term labour as well as primary dysmenorrhea." Regul. Pept. 45:187-191.

Bailey, A. J., and M. L. Rutter. 1991. "Autism." Science Progress 75 (298):389-402.

Barberis, C., and E. Tribollet. 1996. "Vasopressin and oxytocin recetpors in the central nervous system." Crit. Rev. Neurobiol. 10:119-154.

Brownstein, M. J. 1983. "Biosynthesis of vasopressin and oxytocin." Annu Rev. Physiol. 45:129-135.

Carter, C. S. 1992. "Oxytocin and sexual behavior." Neurosci Biobehav. Rev. 16 (131-144).

Chauvet, M T, D Hurpet, J Chauvet, and R Acher. 1983. "Identification of hyman neurophysins complete amino-acid sequences of MSEL and VLDV neurophysins." Proc.Natl. Acad. Sci.USA 80 (10):2839-2843.

Cook, E. H., R. Y. Courchesne, N. J. Cox, C. Lord, D. Gonen, S. J. Guter, A. Lincoln, K. Nix, R. Haas, B. L. Leventhal, and E. Courchesne. 1998. "Linkage-disequilibrium mapping of autistic diosrder with 15q11-13 markers." Am. J. Hum. Genet. 62:1077-1083.

Cool, D. R., and D. DeBrosse. 2003. "Extraction of oxytocin and arginine-vasopressin from serum and plasma for radioimmunoassay and surface enhanced laser desorption-ionization time of flight mass spectrometry." J. Chromatography B 792:375-380.

Corpet, F. 1988. "MULTIPLE SEQUENCE ALIGNMENT WITH HIERARCHICAL CLUSTERING." Nucleic Acids Research 16 (22):1088110890.

Fricker, L. D. 1991. Peptide processing exopeptidases: amino-and carboxy-peptidases involved with peptide biosynthesis. Edited by L.D. Fricker, Peptide biosynthesis and processing. Boca Raton: CRC Press.

Fricker, L. D., Y. L. Berman, E. H. Leiter, and L. A. Devi. 1996. "Carboxypeptidase E activity is deficient in mice with the fat mutation. Effect on peptide processing." J Biol Chem 271 (48):30619-24.

Fricker, L. D., and S. H. Snyder. 1983. "Purification and characterization of enkephalin convertase, an enkephalin-synthesizing carboxypeptidase." J. Biol. Chem. 258 (18):10950-10955.

Fricker, LD. 1988. "Carboxypeptidase E." Annu. Rev. Physiol. 50:309-321.

Green, LeeAnne, Deborah Fein, Charlotte Modahl, Carl Feinstein, Lynn Waterhouse, and Mariana Morris. 2001. "Oxytocin and autistic disorder: Alterations in peptide forms." Biol. Psych. 50 (8):609-613.

Hook, V. Y. 1985. "Differential distribution of carboxypeptidase-processing enzyme activity and immunoreactivity in membrane and soluble components of chromaffin granules." J. Neurochem. 45 (3):987-989.

Hutchens, T. W., and T. T. Yip. 1993. "New desorption strategies for the mass spectrometric analysis of macromolecules." Rapid Commun. Mass Spectrum 7:576-580.

Insel, T. R. 1997. "A neurobiological basis of social attachment." Am. J. Psychiatry 154 (6):726-735.

Insel, T. R., D. J. O'Brien, and J. F. Leckman. 1999. "Oxytocin, vasopressin and autism: is there a connection?" Biol. Psychiatry 45:145-157.

Insel, T.R. 1992. "Oxytocin: a neuropeptide for affiliation-evidence from behavioral, receptor autoradiographic and comparative studies." Psychoneuroendocrinology 17:3-33.

Insel, T.R., and L.E. Shapiro. 1992. "Oxytocin receptor distribution reflects social organization in monogamous and polygamous voles." Proc. Natl. Acad. Sci (USA) 89:5981-5985.

Issaq, H.J., T. D. Veenstra, T. P. Conrads, and D. Felschow. 2002. "The SELDI-TOF MS approach to proteomics: protein profiling and biomarker identification." Biochem. Biophys. Res. Commun. 292:587-592.

Kanner, L. 1943. "Autistic disturbances of affective contact. ." Nervous Child 2:217-250.

Land, H., M. Grez, S. Ruppert, H. Schmale, M. Rehbein, D. Richter, and G. Schutz. 1983. "Deduced amino acid sequence from the bovine oxytocinneurophysin I precursor cDNA." Nature 302:342-344.

Lindberg, I. 1991. "The new eukaryotic precursor processing proteinases." Molecular Endocrinol. 5 (10):1361-1365.

Merchant, M, and S R Weinberger. 2000. "Recent advancements in surface-enhanced laser desorption/ionization-time of flight-mass spectrometry." Electrophoresis 21 (Apr):1164-77.

Modahl, Charlotte, Leeanne Green, Deborah Fein, Mariana Morris, Lynn Waterhouse, Carl Feinstein, and Harriet Levin. 1998. "Plasma oxytocin levels in autistic children." Biological Psychiatry 43 (4):270-277.

Naggert, J. K., L. D. Fricker, O. Varlamov, P. M. Nishina, Y. Rouille, D. F. Steiner, R. J. Carroll, B. J. Paigen, and E. H. Leiter. 1995. "Hyperproinsulinemia in obese fat/fat mice associated with a carboxypeptidase E mutation which reduces enzyme activity." Nature Gen. 10:135-142.

Nishioka, T., J. Anselmo-Franci, P. Li, F. Callahan, and M. Morris. 1998. "Stress increases oxytocin release within the hypothalamic paraventricular nucleus." Brain Research 781:57-61.

Pickles, Andrew, P. Bolton, H. MacDonald, A. Bailey, A. Le Couteur, C.-H. Sim, and M. Rutter. 1995. "Latent-class analysis of recurrence risks for complex phenotypes with selection and measurement error: A twin and family history study of autism." American Journal of Human Genetics 57 (3):717-726.

Samson, W., M. Lumpkin, and S. Mccann. 1986. "Evidence for a physiological role for oxytocin in the control of prolactin secretion." Endocrinology 119:554-560.

Sausville, E., D. Carney, and J. Battey. 1985. "The human vasopressin gene is linked to the oxytocin gene and is selectively expressed in a cultured lung cell line." J. Biol. Chem. 260:10236-10241.

Schopler, E., R.J. Reichler, R.F. DeVellis, and K. Daly. 1980. "Toward objective classification of childhood autism: Childhood Autism Rating Scale (CARS)." Journal of Autism \& Developmental Disorders 10:91-103.

Seidah, NG, L Gaspar, P Mion, M Marcinkiewicz, M Mbikay, and M Chretien. 1990. "cDNA Sequence of two distinct pituitary proteins homologous to Kex 2 and Furin gene products: tissue-specific mRNAs encoding candidates for pro-hormone processing proteinases." $D N A$ and Cell Biol. 9 (6):415-424. 
Steiner, D.F., S.P. Smeekens, S. Ohagi, and S.J. Chan. 1992. "The new enzymology of precursor processing endoproteases." J. Biol. Chem. 267 (33):23435-23438.

Utsunomiya, N., S. Ohagi, T. Sanke, H. Tatsuta, T. Hanabusa, and K. Nanjo. 1998. "Organization of the human carboxypeptidase E gene and molecular scanning for mutations in Japanese subjects with NIDDM or obesity." Diabetologica 41:701-705.

van Wimersma Greidanus, T. B., and C. Maigret. 1996a. "The role of limbic vasopressin and oxytocin in social recognition." Brain Research 713:152-159.

van Wimersma Greidanus, T. B., and C. Maigret. 1996b. "The role of limbic vasopressin and oxytocin in social recognition." Brain Research 713:153-159.

Varlamov, O., E. H. Leiter, and L. Fricker. 1996. "Induced and spontaneous mutations at Ser ${ }^{202}$ of carboxypeptidase E." J. Biol. Chem. 271 (24):13981-13986.

Wing, L. 1997. "The autistic spectrum." Lancet 350:1761-1766.

Wing, L. , and J. Gould. 1979. "Severe impairments of social interaction and associated abnormalities in children: epidemiology and classification." J. Autism Develop. Disorders 9:11-29.

Witt, D. M. 1995. "Oxytocin and rodent sociosexual responses: from behavior to gene expression." Neurosci Biobehav. Rev. 19 (315-324).

Wright, G. L., L. H. Cazares, S. M. Leung, S. Nasim, B. L. Adam, T. T. Yip, P. F. Schellhammer, L. Gong, and A. Vlahou. 1999. "Proteinchip surface enhanced laser desorption/ionization (SELDI) mass spectrometry: a novel protein biochip technology for detection of prostate cancer biomarkers in complex protein mixtures." Prost. Can. Prostat. Dis. 2:264-276.

Young, L. J. 2001. "Oxytocin and Vasopressin as Candidate Genes for Psychiatric Disorders: Lessons from Animal Models." Am. J. Med. Genet. 105:53-54.

Young, Larry J., Miranda M. Lim, Brenden Gingrich, and Thomas R. Insel. 2001. "Cellular mechanisms of social attachment." Hormones and Behavior 40 (2):133-138. 